\title{
Impacto diferenciado del tiempo de formación universitaria según institución de educación media en el desarrollo de habilidades sociales
}

\section{Differentiated Impact of University Training Time According to Secondary Education Institution on the Development of Social Skills}

\author{
Mauricio Salazar Botello \\ Universidad del Bío-Bío, Chillán, Chile \\ ORCID: http://orcid.org/0000-0002-5659-7331 \\ Rodolfo Mendoza-Llanos \\ Universidad del Bío-Bío, Chillán, Chile \\ ORCID: http://orcid.org/0000-0001-9529-570X \\ Yohana Muñoz Jara \\ Universidad del Bío-Bío, Chillán, Chile \\ ORCID: http://orcid.org/0000-0003-3897-8840
}

Recibido 30-11-19 Revisado 12-01-20 Aprobado 13-03-20 En línea 17-03-20

*Correspondencia

Email: rmendoza@ubiobio.cl
Citar como:

Salazar, M., Mendoza-Llanos, R., \& Muñoz, Y. (2020). Impacto diferenciado del tiempo de formación universitaria según institución de educación media en el desarrollo de habilidades sociales. Propósitos y Representaciones, 8(2), e416. doi: http://dx.doi.org/10.20511/pyr2020.v8n2.416 


\section{Resumen}

El desempeño profesional eficiente exige el desarrollo de competencias específicas en conjunto con habilidades sociales. La evidencia indica que existirían diferencias (individuales y contextuales) en el desarrollo de habilidades sociales, sugiriendo que se mantendrían estables en el tiempo. El objetivo del presente estudio fue realizar una comparación de habilidades sociales según sexo, institución educativa de procedencia y años de formación universitaria en 289 estudiantes de una universidad pública del centro-sur de Chile. Los resultados indican que no existen diferencias en habilidades sociales entre hombres y mujeres, pero que estas son mayores entre quienes provienen de instituciones particulares subvencionadas que los de instituciones públicas, así como también entre quienes están en el último año de su formación, por sobre los que cursan primer año. Al agudizarse estas brechas con el paso por la universidad, se transforman en un imperativo de mejora universitario, para generar un desarrollo posterior igualitario entre los estudiantes.

Palabras clave: Desarrollo de habilidades; Relaciones interpersonales; Enseñanza superior; Enseñanza secundaria; Habilidad.

\section{Summary}

Efficient professional performance requires the development of specific competencies in conjunction with social skills. Evidence indicates that there would be differences (individual and contextual) in the development of social skills, suggesting that they would remain stable over time. The aim of the present study was to make a comparison of social skills according to sex, educational institution of origin and years of university formation in 289 students of a public university of the center-south of Chile. The results indicate that there are no differences in social skills between men and women, but that these are greater among those who come from private subsidized institutions than those from public institutions, as well as among those who are in the last year of their training than those who are in the first year. As these gaps widen with the passage through the university, they become an imperative for university improvement, in order to generate a later egalitarian development among students.

Keywords: Skills Development; Interpersonal Relations; Higher Education; Secondary Education, Skills

\section{Introducción}

En una sociedad globalizada y del conocimiento, el desempeño profesional eficiente exige además de las competencias específicas propias del ejercicio de una determinada profesión, competencias genéricas o transversales, tales como: la capacidad de gestionar de forma autónoma y permanente el conocimiento, de investigar, de adaptarse a los cambios, saber relacionarse, de trabajar en equipos, de comunicarse en un segundo idioma y de aprender a lo largo de la vida (ClementeRicolfe \& Escribá-Pérez, 2013; González \& González, 2008; Posada, 2004). El ritmo vertiginoso y complejo propio de la sociedad actual conmina a las personas a interactuar socialmente, en distintas situaciones en las cuales varían las reglas y roles, no siendo tan definidos como en tiempos anteriores y exigiendo a las nuevas generaciones el despliegue correcto y eficaz de sus habilidades sociales (HS) para enfrentarlas (Velásquez et al., 2009).

El contexto educativo universitario no está ajeno a lo anterior, puesto que las HS junto con las exigencias del entorno, se suman a la necesidad del aprendizaje en línea, uso permanente de tecnologías de comunicación que cambian constantemente, generando la necesidad de aprender a aprender, transformándolo en un atributo imperioso en la preparación para la vida de los estudiantes (Bustingorry \& Mora, 2008; Segura, 2002). Países como Estados Unidos, Chile, México, India y China, indican que en un $62 \%, 85 \%, 67 \% 60 \%$ y $27 \%$ respectivamente, la 
educación es muy importante para salir adelante en la vida y destacan que son igual de importante el desarrollo de las habilidades cognitiva como las habilidades interpersonales (Reimers \& Chung, 2016).

Actualmente, la formación no sólo se produce en los sistemas formales, sino que puede lograrse a través de muy diversas fuentes y agentes. La formación universitaria que parte de modelos basados en una concepción del conocimiento y de los contenidos como los objetivos primordiales del aprendizaje está siendo modificada debido al acelerado cambio de los conocimientos, el acceso y la manera en cómo fluye y, en consecuencia, la provisionalidad de los saberes (Irigoyen, Jiménez, \& Acuña, 2011).

Estos cambios paradigmáticos en la formación del profesional de la enseñanza universitaria en el nuevo siglo traen consigo, necesariamente, una concepción diferente de la docencia y de los roles que desempeñan profesores y estudiantes en el proceso de enseñanzaaprendizaje (Irigoyen et al., 2011). De esta manera, la concepción del profesor como transmisor y del estudiante como receptor de conocimientos es sustituida por la concepción del docente como orientador, guía que acompaña al estudiante en el proceso de construcción no sólo de conocimientos, sino también en el desarrollo de habilidades y valores asociados a un desempeño profesional eficiente, ético y responsable, concibiendo la formación y desarrollo de competencias específicas y genéricas en su interrelación en el proceso de formación profesional, lo que ha recibido apoyo en las últimas dos décadas (Contini, 2009; Díaz \& Márquez, 2007; García, 1991; R. González \& González, 2007; V. González \& González, 2008; Hidalgo \& Abarca, 1990; Huambachano \& Huaire, 2018; Reimers \& Chung, 2016; Rosa, Navarro-Segura, \& López, 2014).

Gallego (2008) plantea que las profesiones que tienen como base de actuación, el establecimiento, regulación y mantenimiento de relaciones interpersonales requieren una actuación social competente que puede llegar a ser decisiva en el éxito profesional. Esta afirmación cobra gran importancia para el caso de aquellas formaciones profesionales que consideran la gestión de procesos como un eje principal de su quehacer. Incluso más que definitorio del quehacer profesional, existe evidencia que indica que las HS influyen en la inserción en el mercado laboral, independiente del éxito académico logrado (Herrera et al., 2012; Mendo-Lázaro et al., 2016). Sobre esto último, la evidencia de los últimos años indica una gran preocupación por mejorar las conductas prosociales de los estudiantes universitarios, debido a que los futuros profesionales deben desenvolverse en escenarios complejos (Huambachano \& Huaire, 2018; De Matos et al., 2017; Holst et al., 2017), y al mismo tiempo, las habilidades no cognitivas bajas pueden aumentar los costos no pecuniarios o "psíquicos" de la universidad, disminuyendo así la probabilidad de matriculación (Jacob, 2002).

Se entiende por habilidades sociales (HS) el conjunto de habilidades y capacidades variadas como específicas para el contacto interpersonal y la situación de problemas de índole personal, así como socioemocional, desde actividades de carácter básico hacia otras de características avanzadas e instrumentales (Goldstein, Sprafkin, Gershaw, \& Klein, 1980). La conducta socialmente hábil es ese conjunto de conductas emitidas por un individuo en un contexto interpersonal que expresa los sentimientos, necesidades, actitudes, preferencias, deseos, opiniones o derechos de ese individuo de un modo adecuado a la situación, sin ansiedad excesiva y de manera no aversiva, respetando esas conductas en los demás, y que trae como consecuencia el auto-reforzamiento y maximiza la probabilidad de conseguir refuerzo externo (Caballo, 1986, 2007; Gismero, 1996).

Para Zavala, Valadez y Vargas (2008) las HS son conductas observables, aprendidas y utilizadas en los intercambios sociales para obtener fines concretos, por lo que son susceptibles de cambiar, y que por tanto juegan un rol preponderante en la capacidad individual de enfrentar los desafíos de la edad adolescente, contribuyendo en la aceptación social. Esto se podría ver acrecentado en el paso por la universidad dado que es un momento en el cual los adolescentes todavía están en la formación de su identidad (Barrera-Herrera \& Vinet, 2017). 
Existe evidencia en distintos ámbitos de la literatura científica que da cuenta de la importancia de las HS en el éxito o fracaso de las personas al desenvolverse en sociedad (Huambachano \& Huaire, 2018; Herrera et al., 2012). Algunos reportes indican que están relacionadas con el clima social familiar $(r=, 39 ; p<, 001$; García Nuñez Del Arco, 2005) o con experiencias durante la adolescencia (Oyarzún, Estrada, Pino, \& Oyarzún, 2012; Zavala et al., 2008), lo que acompaña el paso por la educación media. Aunque se ha encontrado evidencia a favor de la correlación entre rendimiento académico con HS (Oyarzún et al., 2012) y en contra $(r$ $=-, 02 ; p>, 05 ;$ García Nuñez Del Arco, 2005). Dada la importancia del paso por la universidad (Barrera-Herrera y Vinet, 2017), es probable que las habilidades no cognitivas influyan posteriormente en el empleo, la elección de ocupación y una variedad de otros resultados del mercado laboral, existiendo incluso un planteamiento de que las HS tienen un efecto que es comparable en magnitud al estatus socioeconómico (Jacob, 2002).

Oyarzún et al. (2012) en una muestra de adolescentes, encontraron que no existía relación entre rendimiento académico y HS, pero si encontraron perfiles diferentes por sexo, reportando que en mujeres, el rendimiento académico correlaciona marginalmente con HS $(r=, 13 ; p=, 059)$, y concluyen que, en mujeres, el rendimiento tiende a asociarse positivamente con la presencia de HS por parte de los otros. En varones, no encontraron relación. Estos autores (Oyarzún et al., 2012) plantean que las diferencias por sexo observadas en la edad escolar tienden a mantenerse durante la formación universitaria, y que el desarrollo deficiente de HS durante el periodo escolar no puede ser "reparado" durante la formación universitaria, manteniendo su efecto diferencial en el rendimiento académico. Estas diferencias y rendimiento superior de las mujeres podrían deberse a características propias de las mujeres, entre ellas mayores HS (Echavarri, Godoy, \& Olaz, 2007). Para Herrera et al. (2012) los estudiantes ingresan a la universidad con un repertorio conductual deficitario que se acrecienta al estar próximos a finalizar su carrera, lo que podría indicar que, al no contar con instancias formales de entrenamiento, las HS podrían inhibirse paulatinamente.

Desde una perspectiva psicométrica, Morán y Olaz (2014) realizaron una revisión bibliométrica sobre instrumentos para evaluar HS - y constructos afines - en América Latina entre los años 1990 al 2013, concluyendo que los reportes son aislados, en poblaciones de niños y estudiantes universitarios; con escazo reporte de propiedades psicométricas (centradas en confiabiliad y análisis factorial exploratorio). Estudios posteriores responden a estos cuestionamientos metodológicos con propuestas específicas para HS para muestras latinoamericanas y específicamente para el caso de Chile (Miranda-Zapata, Riquelme-Mella, Cifuente-Cid, \& Riquelme-Bravo, 2014) o desarrollos posteriores (Caballo et al., 2017). En el mejor de nuestro conocimiento la Escala de Habilidades Sociales (EHS) de Gismero (2002) es un instrumento permanentemente utilizado en la investigación sobre HS en América latina (MirandaZapata et al., 2014; Zavala et al., 2008).

La primera aproximación se realiza observando el análisis realizado por Miranda-Zapata et al. (2014) para la EHS de Gismero (2000). En su estudio realizado en una muestra de 1206 estudiantes universitarios del sur de Chile obtuvieron una confiabilidad global adecuada $(\alpha=, 91)$ y para sus dimensiones: Autoexpresión en situaciones sociales (ASS); Defensa de los propios derechos como consumidor (DDC); Expresión de enfado o disconformidad (DIS); Decir no y cortar interacciones (NO); Hacer peticiones (HP); Iniciar interacciones positivas con el sexo opuesto (IPO).

En atención a lo planteado anteriormente, el presente estudio tiene por objetivo determinar el efecto que tienen tres variables en el desarrollo de las HS en estudiantes de una universidad pública del centro sur del Chile. El sexo, el tipo de institución educativa de procedencia y el momento en el que son evaluadas las habilidades sociales. Como objetivos específicos se busca: (1) determinar el grado de relación entre edad y HS; (2) determinar 
diferencias según sexo o institución de procedencia sobre las HS; y (3) determinar diferencias entre los años de formación universitaria recibida sobre las HS.

Aunque el objetivo de este estudio no fue un análisis psicométrico de la Escala de Habilidades Sociales per se, se detallan a continuación algunos indicadores para aportar a los resultados presentados por Miranda-Zapata et al. (2014), en atención a las críticas específicas que plantea Caballo et al. (2017) respecto de la EHS.

\section{Método}

La presente investigación corresponde a un estudio transversal, correlacional. Participaron 289 estudiantes de dos carreras asociadas a las ciencias empresariales de una universidad pública del centro sur de Chile, de edad promedio 20,71 años $(D S=3,72)$, de los cuales el 58,13\% eran mujeres; de establecimientos educacionales de procedencia pública $(41,52 \%)$ y particular subvencionados $(58,48 \%)$. La selección de los participantes fue no probabilística, por conveniencia, cuyo criterio de selección correspondía a la pertenencia a primer $(n=164)$ o último $(\mathrm{n}=125)$ año de carrera que cursaba.

\section{Instrumentos}

Se utilizó la Escala de Habilidades Sociales de Gismero (2000) en su versión original, que contiene 33 ítems en formato tipo likert donde $\mathrm{A}=$ "No me identifico en lo absoluto; la mayoría de las veces no me ocurre o no lo haría", B = "Más bien no tiene que ver conmigo, aunque alguna vez me ocurra", $\mathrm{C}=$ "Me describe aproximadamente, aunque no siempre actúe o me sienta así" y D = "Muy de acuerdo y me sentiría o actuaría así en la mayoría de los casos". Contiene seis dimensiones: Autoexpresión en situaciones sociales ( 8 ítems); Defensa de los propios derechos como consumidor (6 ítems); Decir no y cortar interacciones (6 ítems); Hacer peticiones (5 ítems); Iniciar interacciones positivas con el sexo opuesto (5 ítems); Expresión de enfado o disconformidad (3 ítems), para los cuales Miranda-Zapata et al. (2014) reportan una confiabilidad de, $83 ;, 62 ;, 72 ;, 37 ;, 48$ y ,39 respectivamente, y un $\alpha_{\text {global }}=, 91$, en una solución que incorpora una dimensión de segundo orden.

Además, se consideran preguntas sociodemográficas como sexo, edad, curso inicial o final, y tipo de institución educativa de procedencia (pública o particular subvencionada).

\section{Procedimiento}

El instrumento se aplicó en dos carreras pertenecientes a las Ciencias Empresariales. La aplicación se realizó voluntariamente durante el primer semestre académico (marzo-julio), previa firma del consentimiento informado velando por el cumplimiento de los principios éticos: respeto de la autonomía; no-maleficencia; beneficencia y justicia (Osorio, 2000). La encuesta se aplicó de manera colectiva, previa autorización de los encargados de las carreras.

\section{Análisis de datos}

Se realizó un Análisis Factorial Confirmatorio (AFC) mediante Ecuaciones Estructurales, siguiendo la propuesta de 6 factores y un factor general de segundo orden planteado por MirandaZapata et al. (2014) quienes lo plantean como un avance a la solución factorial propuesta por Gismero (2000).

En el AFC el modelo fue estimado mediante Mínimos Cuadrados No Ponderados (ULS) por tratarse de variables ordinales, y como una manera de mantener el método utilizado por Gismero (2000) y Miranda-Zapata et al. (2014). La bondad de ajuste global del modelo fue evaluada mediante el indicador $\chi^{2} / \mathrm{gl}$, Índice de Ajuste Global (GFI), el Índice de Tucker- Lewis (TLI), el Índice de Ajuste Comparativo (CFI), y el Error Cuadrático Medio de Aproximación 
(RMSEA). Se espera valores cercanos a 2 para el indicador $\chi^{2} / \mathrm{gl}$; valores de GFI, TLI y CFI superiores a 0,90 y 0,95 , y valores de RMSEA menores a 0,08 y 0,05 , se consideran aceptables y buenos respectivamente (Schermelleh-Engel, Moosbrugger, \& Müller, 2003). Para evaluar confiabilidad se calcularon: Omega de McDonald, alfa de Cronbach, sus intervalos de confianza y correlación interitem de acuerdo a Domínguez (2012).

Para responder a los objetivos del estudio se realizan análisis de correlación y comparación de medias mediante la prueba $t$ de Student y su respectivo cálculo de tamaño del efecto mediante $d$ de Cohen. Para interpretar las correlaciones se utilizan los criterios de Gignac y Szodorai (2016) quienes sugieren que la interpretación de las correlaciones en estudios sociales correspondería a pequeña, mediana y grande con índices de, $15 ;, 25$ y ,35 respectivamente. Para la interpretación de tamaño del efecto se utilizan los propuestos en Erceg-Hurn y Mirosevich (2008) donde ,20; ,50 y ,80 se interpretan como efectos pequeño, mediano y grande respectivamente.

Todos los análisis se realizaron mediante el software JASP 0.11 (JASP Team, 2019).

\section{Resultados}

Al realizar el AFC de acuerdo al modelo 2 propuesto por Miranda-Zapata et al. (2014), se obtienen indicadores de ajuste factorial buenos del modelo propuesto $\left(\chi^{2}=544,83 ; g l=489 ; p>, 05 ; \chi^{2} / g l\right.$ $=1,11 ; \mathrm{GFI}=, 99$; TLI $=, 99 ; \mathrm{CFI}=, 99$; RMSEA $=, 02)$, por lo que su solución factorial es aceptable.

Respecto de la confiablidad del instrumento, los resultados (Tabla 1) indican que de manera global es adecuada $(\alpha=, 88)$. Respecto de los seis subdimensiones que lo componen, dos subescalas (ASS e IPO) presentan confiabilidad adecuada; NO se encuentra cercano al criterio estándar $(\alpha>, 7)$; sin embargo, DDC, DIS y HP presentan una baja confiabilidad.

Tabla 1.

Confiabilidad de la escala de Habilidades Sociales

\begin{tabular}{|c|c|c|c|c|c|c|}
\hline & \multirow[b]{2}{*}{ ítem } & \multirow[b]{2}{*}{$\omega$} & \multirow[b]{2}{*}{$\alpha$} & \multicolumn{2}{|c|}{ IC $95 \% \alpha$} & \multirow[b]{2}{*}{ Correlación interitem } \\
\hline & & & & Inferior & Superior & \\
\hline $\mathrm{HS}$ & 33 & ,88 & ,88 &, 86 & ,90 &, 18 \\
\hline ASS & 8 & ,76 & ,75 &, 71 &, 79 & ,28 \\
\hline DDC & 6 & ,57 &, 55 & ,46 & ,62 &, 17 \\
\hline NO & 6 & ,68 & ,67 & 61 & ,63 & ,26 \\
\hline $\mathrm{HP}$ & 5 & ,43 & ,42 & ,31 &, 52 & ,12 \\
\hline IPO & 5 & ,75 & ,74 & ,69 &, 79 & ,37 \\
\hline DIS & 3 &, 55 & ,54 & ,44 & ,63 & ,28 \\
\hline
\end{tabular}

$\mathrm{n}=289$. ASS $=$ Autoexpresión en situaciones sociales; $\mathrm{DDC}=$ Defensa de los propios derechos como consumidor; $\mathrm{NO}=$ Decir no y cortar interacciones; HP=Hacer peticiones; IPO=Iniciar interacciones positivas con el sexo opuesto; DIS=Expresión de enfado o disconformidad

A pesar de estos resultados obtenidos en confiabilidad de subdimensiones, pero considerando que la estructura factorial tiene un buen ajuste y que la confiabilidad global del instrumento da cuenta del constructo general de HS, al cubrir de mejor forma el contenido del constructo que se entiende multidimensional (Oyanedel, Vargas, Mella \& Páez, 2017), se decide continuar con el reporte y los objetivos explicativos planteados para esta investigación, y reportar sus análisis para aquellas subdimensiones que obtuvieron una confiabilidad adecuada (Autoexprepresión en situaciones sociales, Decir no y Cortar interacciones e Iniciar interacciones positivas con el sexo opuesto) (ver Tabla 2). 
Tabla 2.

Estadísticos descriptivos generales y correlaciones según sexo

\begin{tabular}{|c|c|c|c|c|c|c|c|c|c|c|c|c|}
\hline & $M$ & $D S$ & Min & Max & Edad & HS & ASS & DDC & $\mathrm{NO}$ & HP & IPO & DIS \\
\hline Edad & 20,71 & 3,72 & 18 & 41 & - & $23 * *$ &, $17 *$ &, $18 *$ &, 13 &, 15 & $20 * *$ & $25 * *$ \\
\hline HS & 96,90 & 14,86 & 55 & 126 &, 10 & - &, $87 * * *$ &, $73 * * *$ &, $74 * * *$ &, $67 * * *$ &, $77 * * *$ &, $71 * * *$ \\
\hline ASS & 23,76 & 4,71 & 10 & 32 & ,04 &, $81 * * *$ & - &, $50 * * *$ &, $47 * * *$ &, $51 * * *$ &, $72 * * *$ &, $48 * * *$ \\
\hline DDC & 17,66 & 3,29 & 10 & 24 & ,04 &, $74 * * *$ &, $48 * * *$ & - &, $51 * * *$ &, $35 * * *$ &, $40 * * *$ &, $60 * * *$ \\
\hline $\mathrm{NO}$ & 18,27 & 3,58 & 9 & 24 & ,13 &, $76 * * *$ &, $53 * * *$ &, $48 * * *$ & - &, $42 * * *$ &, $40 * * *$ &, $57 * * *$ \\
\hline HP & 15,02 & 2,80 & 6 & 20 &,- 04 &, $51 * * *$ &, $25 * *$ &, $35 * * *$ &, $29 * *$ & - &, $41 * * *$ &, $43 * * *$ \\
\hline IPO & 13,25 & 3,67 & 5 & 20 &, 10 &, $71 * * *$ &, $55 * * *$ &, $35 * * *$ &, $42 * * *$ &, $20 *$ & - &, $37 * * *$ \\
\hline DIS & 8,94 & 2,08 & 3 & 12 &, $20 *$ &, $61 * * *$ &, $44 * * *$ &, $40 * * *$ &, $44 * * *$ & , 13 &, $33 * * *$ & - \\
\hline
\end{tabular}

$* \mathrm{p}<.05, * * \mathrm{p}<.01, * * * \mathrm{p}<.001 ;$ ASS= Autoexpresión en situaciones sociales; DDC= Defensa de los propios derechos como consumidor; $\mathrm{NO}=$ Decir no y cortar interacciones; HP=Hacer peticiones; IPO=Iniciar interacciones positivas con el sexo opuesto; DIS=Expresión de enfado o disconformidad. Bajo diagonal hombres; sobre la diagonal mujeres.

En términos generales y sin diferenciar por sexo, a medida que la edad aumenta, mejoran las habilidades sociales $(r=, 17 ; p<, 01)$; así como también la expresión de enfado o disconformidad $(r=, 22 ; p<, 001)$; iniciar interacciones positivas con el sexo opuesto $(r=, 16 ; p$ $<, 01)$; y decir que no y cortar interacciones $(r=, 13 ; p<, 05)$. Sin embargo, estas asociaciones varían según sexo (Tabla 2). Al observar las correlaciones por sexo, solo es estadísticamente significativa y positiva la asociación de edad y HS, autoexpresión en situaciones sociales, defensa de sus propios derechos como consumidor en las mujeres.

Para corroborar las asociaciones anteriores, al comparar por sexo (Tabla 3) no se observan diferencias significativas entre mujeres y hombres.

Tabla 3.

Comparación de medias entre mujeres y hombres

\begin{tabular}{lcccccc}
\hline & Sexo & $M$ & $S D$ & $t$ & $p$ & $d$ \\
\hline HS* & mujeres & 96.81 & 15.96 & $-0,12$ & 0,91 & $-0,01$ \\
& hombres & 97.02 & 13.25 & & & \\
ASS* & mujeres & 23.45 & 5.24 & $-1,30$ & 0,19 & $-0,15$ \\
& hombres & 24.18 & 3.85 & & & \\
NO & mujeres & 18.47 & 3.73 & 1,14 & 0,25 & 0,14 \\
& hombres & 17.98 & 3.34 & & & \\
IPO & mujeres & 13.08 & 3.76 & $-0,89$ & 0,38 & $-0,11$ \\
& hombres & 13.47 & 3.54 & & & \\
\hline
\end{tabular}

Mujeres $=168 ;$ hombres $=121 ; g l=287 ; *$ No se han asumido varianzas iguales

Como se observa en la Tabla 4, existen diferencias significativas entre los estudiantes al comparar su institución de procedencia (público o particular subvencionado), en HS siendo mayores en aquellos alumnos con procedencia de instituciones particular subvencionado que en municipalizados $(t=-1,95 ; p=, 05 ; d=-0,23)$. 
Tabla 4.

Comparación de medias de habilidades sociales de acuerdo a institución de origen

\begin{tabular}{llccccc}
\hline \multicolumn{1}{c}{ Institución de origen } & $M$ & $S D$ & $t$ & $p$ & $d$ \\
\hline \multirow{2}{*}{ HS } & Público & 94,88 & 14,99 & $-1,95$ & 0,05 & $-0,23$ \\
& Particular Subvencionado & 98,33 & 14,64 & & & \\
\multirow{2}{*}{ NSS } & Público & 23,71 & 4,78 & $-0,15$ & 0,88 & $-0,02$ \\
& Particular Subvencionado & 23,79 & 4,68 & & & \\
& Público & 18,03 & 3,71 & $-0,93$ & 0,35 & $-0,11$ \\
& Particular Subvencionado & 18,43 & 3,48 & & & \\
& Público & 12,88 & 3,76 & $-1,45$ & 0,15 & $-0,17$ \\
& Particular Subvencionado & 13,51 & 3,58 & & & \\
\hline
\end{tabular}

Público $=120 ;$ Particular subvencionado $=169 ; g l=287$. ASS $=$ Autoexpresión en situaciones sociales; $\mathrm{NO}=$ Decir no y cortar interacciones; IPO=Iniciar interacciones positivas con el sexo opuesto

Al comparar HS entre los estudiantes de primer y último año de carrera (Tabla 5) se observan diferencias estadísticamente significativas en favor de los últimos con tamaño de efecto medio ( $t=-2,06 ; p=, 04 ; d=-0,25)$; y cercano a la significancia estadística iniciar interacciones positivas con el sexo opuesto $(t=-1,80 ; p=, 07 ; d=-0,21)$.

Tabla 5.

Comparación de medias de habilidades sociales de acuerdo a año de estudio

\begin{tabular}{lcccccc}
\hline & Año de estudio & $M$ & $S D$ & $t$ & $p$ & $d$ \\
\hline HS & Primero & 95,33 & 15,50 & $-2,06$ & 0,04 & $-0,25$ \\
& Último & 98,95 & 13,77 & & & \\
\multirow{2}{*}{ ASS } & Primero & 23,51 & 4,90 & $-1,04$ & 0,30 & $-0,12$ \\
& Último & 24,09 & 4,45 & & & \\
\multirow{2}{*}{ NO } & Primero & 17,98 & 3,56 & $-1,59$ & 0,11 & $-0,19$ \\
& Último & 18,65 & 3,57 & & & \\
\multirow{2}{*}{ IPO* } & Primero & 12,91 & 3,91 & $-1,80$ & 0,07 & $-0,21$ \\
& Último & 13,69 & 3,28 & & & \\
\hline
\end{tabular}

Primero $=164 ;$ Último $=125 ; g l=287 ; *$ No se han asumido varianzas iguales. ASS $=$ Autoexpresión en situaciones sociales; $\mathrm{NO}=$ Decir no y cortar interacciones; IPO=Iniciar interacciones positivas con el sexo opuesto

Los resultados en las comparaciones con las distintas variables consideradas determinantes dan cuenta que no existen diferencias entre sexos, pero si existen diferencias en los puntajes de las variables HS, dependiendo de la institución de procedencia y año que cursa de carrera, por lo que se realizó una ANOVA 2X2 para evaluar posibles efectos de interacción. Cuando la interacción centro de educación de procedencia y año de carrera universitaria que cursa resulta significativa se examinan los efectos simples.

El ANOVA 2X2 para HS permite observar un efecto principal marginalmente significativo para institución de procedencia, $F(1,285)=3,54 ; p=06 ; \eta^{2}=0,01$, y no significativo para año de formación, $F(1,285)=2,83 ; p=, 09 ; \eta^{2}=0,01$, ni para efecto de interacción entre institución de procedencia y año de formación, $F(1,285)=0,71 ; p=, 40 ; \eta^{2}<$ 0,01 . Al examinar los efectos principales simples se observa que existe mayor puntaje en HS en estudiantes de pertenencia a instituciones particulares subvencionadas de último año que los de primero $(F=3,99 ; p=, 05)$, lo que no se replica entre estudiantes de primer y último año 
provenientes de instituciones públicas $(F=0,29 ; p=, 59)$, lo que se puede observar en la Figura 1.

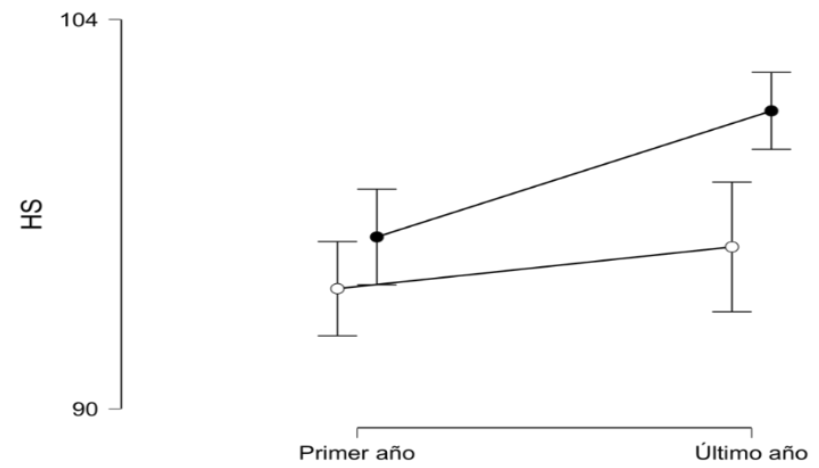

Figura 1. Comparación puntajes de HS según institución de procedencia y año que cursa en la universidad.

\section{Discusión}

En términos de propiedades psicométricas, los resultados obtenidos aquí son similares a los presentados por Miranda-Zapata et al. (2014), puesto que la EHS presenta una confiabilidad global similar en ambas muestras $(\alpha>, 85)$ e indicadores de estructura factorial adecuados. Miranda-Zapata et al (2014) concluyen que el instrumento es una escala fiable y cuyo modelo estructural original se ajusta a la muestra utilizada, aunque es necesario considerar que los índices de fiabilidad por subdimensión - y solo en algunos casos - presentan valores bajos (DDC, HP y DIS), recomendando el instrumento para la evaluación de las habilidades sociales en estudiantes universitarios chilenos. Nuestros resultados son similares a los obtenidos por Miranda-Zapata et al. (2014), los que aun cuando mantienen bajos índices de confiabilidad, aportan - de acuerdo a la confiabilidad general - a mantener el constructo de HS. A nuestro entender las subdimensiones mencionadas (DDC, HP y DIS) deben utilizarse con precaución, -dado que sus confiabilidades son débiles-, mientras no se avance en el desarrollo de más estudios que puedan aportar una mejora en ellas.

En términos generales, nuestros resultados indican que la relación entre edad y HS varía de manera distinta entre hombres y mujeres, siendo solo para estas últimas una relación positiva de intensidad media $(r=, 23 ; p<, 01)$, sin embargo, no se encontraron diferencias significativas en los puntajes de HS según sexo. Si bien estos resultados difieren de estudios previos (Oyarzún et al., 2012; Echavarri et al., 2007), se observa un desarrollo de tendencia positiva en las mujeres con el paso del tiempo que podría "reparar" el desarrollo deficiente de HS, igualándolas en el tiempo, contraviniendo la afirmación de estabilidad de las HS planteada por Oyarzún et al. (2012).

Respecto de la institución de procedencia sobre las HS, nuestros resultados indican que quienes estudiaron en instituciones particulares subvencionadas, presentan mayores puntajes en HS que quienes vienen de instituciones municipales. Esta evidencia puede estar relacionada con otros elementos como el clima familiar (García Nuñez Del Arco, 2005) y otras experiencias adolescentes positivas (Oyarzún et al., 2012; Zavala et al., 2008) que pueden tener su explicación en la desigualdad que se ha arrastrado en los centros educacionales dependiendo del origen de su financiamiento (García-Huidobro y Belleï, 2003).

Finalmente, y de acuerdo al año de formación que cursan los estudiantes, el paso por la universidad tiende a acrecentar las diferencias en puntajes de HS con un tamaño de efecto medio $(d=, 25)$ favoreciendo el desarrollo de competencias no cognitivas deseables para el desempeño 
posterior en el mundo laboral (Velásquez et al., 2009, Irigoyen et al., 2011). Estos resultados pueden estar asociado a otras variables como estrés por no conocer las dinámicas y tiempos universitarios, por ejemplo, es conocido el valor como factor protector de las habilidades sociales sobre este respecto. Esto nos lleva a suponer que existe un fortalecimiento de las habilidades socioemocionales en los estudiantes, en general, independiente de su sexo. Aprenden a manejar la presión y estrés que significa enfrentar la vida universitaria en los primeros años y esto está asociado a la experiencia que se construye en el tránsito hacia los últimos años de carrera.

Sin embargo, los efectos entre el año que cursan los estudiantes (primero y último) se observan diferencias de acuerdo a la institución de origen, no favoreciendo, de esta forma, el planteamiento de Jacob (2002) de permitir compensar a quienes tienen un estatus socioeconómico más bajo. Los resultados asociados al tipo de institución educacional de procedencia, nos indica que inicialmente existe una brecha en las habilidades sociales entre los estudiantes que provienen de instituciones públicas y colegios particulares subvencionados. Los estudiantes de instituciones públicas en primer año de universidad muestran habilidades sociales más bajas que los estudiantes de colegios particulares subvencionados. Esta diferencia o brecha se mantiene en los últimos años de universidad e incluso se acrecienta, los estudiantes de colegios particular subvencionados que inicialmente muestran mayores habilidades sociales, las mantienen $y$ aumentan considerablemente en los últimos años de universidad.

A nuestro modo de ver, el desarrollo de HS en conjunto con saberes y competencias técnicas es complejo de trabajar con los estudiantes, pues para estos últimos en muchos casos las HS no son centrales - que en conjunto con un sistema educativo escolarizante - no alcanza a relevar los requerimientos del mundo laboral en estos aspectos (Vargas, 2017; Sandberg, 2000). Por cierto, esto no es responsabilidad exclusiva de los estudiantes, pues los planes de estudio podrían eventualmente propiciar desde su inicio un contacto mayor con el mundo del trabajo, en tal caso las prioridades podrían cambiar antes de los últimos años (Tobón, 2013). Esto no significa que las universidades no realicen esfuerzos por abordar estos temas, pero al parecer, las iniciativas existentes son insuficientes, toda vez que carecen de sistematización, continuidad y seguimiento. Además, el desarrollo de habilidades sociales, sin duda, es una responsabilidad que también responde a un imperativo académico transversal que atañe a los profesores, quienes requieren también preparación y formación sistémica para entregar y ayudar a los estudiantes en su formación personal y profesional (Villarroel \& Bruna, 2014).

Desde una perspectiva más casuística, los resultados ratifican nuestra experiencia práctica como académicos, donde observamos que los estudiantes se ven más desenvueltos en los últimos años y son capaces de ser más asertivos en diferentes aspectos, en algunos casos esto se transforma para ellos en una necesidad que desean trabajar, pero se dan cuenta que ya ha pasado tiempo y éste se ha perdido. Sin embargo, entendemos que las HS se puedan trabajar en todo momento y siempre se pueden mejorar.

Todas las acciones que se puedan realizar respecto de desarrollar habilidades sociales serán un factor diferenciador en el ejercicio del rol profesional futuro, y también en la mantención de la salud mental de los estudiantes. Todos los estudios longitudinales que se puedan realizar para validar esto - y entregar apoyo empírico - que corroboren que la exposición al ámbito universitario mejora las HS podrían generar una mirada nueva respecto de la conceptualización de lo que significa el curriculum universitario, siendo pertinente intervenir en HS de manera diferenciada de acuerdo con Oyarzún et al. (2012) considerando la institución de procedencia durante el periodo de formación universitaria. Esta última recomendación cobra sentido social, si la formación universitaria quiere cumplir como rol social la búsqueda de oportunidades de desarrollo igualitarias a todos sus egresados, nivelando hacia lo esperado en un profesional integral.

Sin duda este trabajo es una primera aproximación para entender cuán importante es el paso por la universidad para el desarrollo de las habilidades sociales y la relevancia que posee 
diferenciar el currículo para atender los disímiles requerimientos de los estudiantes considerando su procedencia. Esto no resulta tan fácil ya que las muestras universitarias tienen sus propias particularidades (Arnett, 2004). Sin embargo, abre un espacio para profundizar una línea de investigación en docencia.

\section{Referencias}

Arnett, J. J. (2004). Emerging adulthood: The winding road from the late teens through the twenties. New York: Oxford University Press.

Barrera-Herrera, A., \& Vinet, E. (2017). Adultez Emergente y características culturales de la etapa en universitarios chilenos. Terapia Psicológica, 35(1), 47-56.

Bustingorry, S. O., \& Mora, S. J. (2008). Metacognición: Un camino para aprender a aprender. Estudios Pedagogicos, 34(1), 187-197. doi: https://doi.org/10.4067/S071807052008000100011

Caballo, V. (1986). Evaluación de las Habilidades Sociales. Madrid: Pirámide.

Caballo, V. (2007). Manual de evaluación y entrenamiento de las habilidades sociales (7th ed.). España: Siglo XXI Editores.

Caballo, V., Salazar, I. C., \& CISO-A, E. de I. (2017). Desarrollo y validación de un nuevo instrumento para la evaluación de las habilidades sociales: el "Cuestionario de habilidades sociales" (CHASO). Psicología Conductual, 25(1), 5-24.

Clemente-Ricolfe, J., \& Escribá-Pérez, C. (2013). Análisis de la percepción de las competencias genéricas adquiridas en la universidad. Revista de Educación, (362), 535-561. doi: https://doi.org/10.4438/1988-592X-RE-2013-362-241

Contini, N. (2009). Las habilidades sociales en la adolescencia temprana perspectivas desde la Psicología Positiva. Psicodebate. Psicología, Cultura y Sociedad, (9), 45-64.

De Matos, D., Bruna, H., \& Vera, J. Á. (2017). Habilidades sociales y autoeficacia en la formación superior. Revista Intercontinental de Psicología y Educación, 19(1-2), 113-134.

Díaz, J., \& Márquez, J. (2007). Estimación del potencial de competencias en la implementación de ejes transversales en instituciones de Educación Superior. Sapiens: Revista Universitaria de Investigación, 205-224.

Domínguez, S. (2012). Propuesta para el cálculo del Alfa Ordinal y Theta de Armor. Revista de Investigación en Psicología, 15(1), 213-217.

Echavarri, M., Godoy, J. C., \& Olaz, F. (2007). Diferencias de género en habilidades cognitivas y rendimiento académico en estudiantes universitarios. Universitas Psychologica, 6(2), 319-329.

Erceg-Hurn, D. M., \& Mirosevich, V. M. (2008). Modern Robust Statistical Methods: An Easy Way to Maximize the Accuracy and Power of Your Research. American Psychologist, 63(7), 591-601. doi: https://doi.org/10.1037/0003-066X.63.7.591

Gallego, Ó. (2008). Descripción de las habilidades sociales en estudiantes de psicología de una institución de educación superior. Revista Iberoamericana de Psicología: Ciencia y Tecnología, (1), 61-71.

García, J. (1991). La formación integral: Objetivo de la universidad (algunas reflexiones sobre la educación en la universidad). Revista Complutense de Educación, 2(2), 323-335.

García-Huidobro, J. y Belleï, C. (2003). Desigualdad Educativa en Chile. Universidad Alberto Hurtado, Santiago: Chile

García Nuñez Del Arco, C. (2005). Habilidades sociales, clima social familiar y rendimiento académico en estudiantes universitarios. Liberabit. Revista Peruana de Psicología, 11, $63-$ 74.

Gignac, G. E., \& Szodorai, E. T. (2016). Effect size guidelines for individual differences researchers. Personality and Individual Differences, 102(November 2016), 74-78. doi: https://doi.org/10.1016/j.paid.2016.06.069

Gismero, E. (1996). Habilidades Sociales y anorexia nerviosa. Madrid: Publicaciones Universidad Pontificia Comillas.

Goldstein, A., Sprafkin, R., Gershaw, J., \& Klein, P. (1980). Habilidades sociales y autocontrol en la adolescencia. España: Ediciones Martínez Roca. 
González, R., \& González, V. (2007). Diagnóstico de necesidades y estrategias de formación docente en las Universidad. Revista Iberoamericana de Educación, 43(6).

González, V., \& González, R. (2008). Competencias genéricas y formación profesional: un análisis desde la docencia universitaria. Revista Iberoamericana de Educación, (47), 185209.

Herrera, A., Freytes, V., López, G., \& Olaz, F. (2012). Un estudio comparativo sobre las habilidades sociales en estudiantes de psicología. International Journal of Psychology and Psychological Therapy, 12(2), 277-287.

Hidalgo, C., \& Abarca, N. (1990). Desarrrollo de habilidades sociales en estudiantes universitarios. Revista Latinoamericana de Psicología, 22(2), 265-282.

Holst, I., Galicia, Y., Gómez, G., \& Degante, A. (2017). Las habilidades sociales y sus diferencias en estudiantes universitarios. Revista Especializada en Ciencias de la Salud, 20(2), 22-29.

Huambachano, A. M., \& Huaire, E. (2018). Desarrollo de habilidades sociales en contextos universitarios. Horizonte de la Ciencia, 8(14), 123-130. doi: https://doi.org/10.26490/uncp.horizonteciencia.2018.14.430

Irigoyen, J., Jiménez, M., \& Acuña, K. (2011). Competencias y Educación Superior. Revista Mexicana de Investigación Educativa (RMIE), 16(48), 243-266.

Jacob, B. A. (2002). Where the boys aren't: non-cognitive skills, returns to school and the gender gap in higher education. Economics of Education Review, 21, 589-598.

JASP Team (2019). JASP (Version 0.11) [Computer software].

Mendo-Lázaro, S., León, B., Felipe-Castaño, E., Polo, M. I., \& Palacios-García, V. (2016). Evaluación de las habilidades sociales de estudiantes de Educación Social. Revista de Psicodidactica, 21(1), 139-156. doi: https://doi.org/10.1387/RevPsicodidact.14031

Miranda-Zapata, E., Riquelme-Mella, E., Cifuente-Cid, H., \& Riquelme-Bravo, P. (2014). Análisis factorial confirmatorio de la Escala de Habilidades Sociales en universitarios chilenos. Revista Latinoamericana de Psicología, 46(2), 73-82. doi: https://doi.org/10.1016/S0120-0534(14)70010-X

Morán, V., \& Olaz, F. (2014). Instrumentos de evaluación de habilidades sociales en América Latina: un análisis bibliométrico. Revista de Psicología (Santiago), 23(1), 93-105. doi: https://doi.org/10.5354/0719-0581.2014.32877

Osorio, J. (2000). Principios éticos de la investigación en seres humanos y en animales. Medicina, 60(2), 255-258.

Oyanedel, J. C., Vargas, S., Mella, C., \& Páez, D. (2017). Cálculo de confiabilidad a través del uso del coeficiente Omega de McDonald. Revista Médica de Chile, 145(2), 272-273. doi: doi.org/10.4067/S0034-98872017000200018

Oyarzún, G., Estrada, C., Pino, E., \& Oyarzún, M. (2012). Habilidades sociales y rendimiento académico: una mirada desde el género. Acta Colombiana de Psicología, 15(2), 21-28.

Posada, R. (2004). Formación superior basada en competencias, interdisciplinariedad y trabajo autónomo del estudiante. Revista Iberoamericana de Educación, 1-33.

Reimers, F., \& Chung, C. (2016). Enseñanza y aprendizaje en el siglo XXI: Metas, políticas educativas y currículo en seis países (p. 325). p. 325. Ciudad de México: Fondo de Cultura Económica.

Rosa, G., Navarro-Segura, L., \& López, P. (2014). El aprendizaje de las habilidades sociales en la Universidad. Análisis de una experiencia formativa en los grados de educación social y trabajo social. Formación Universitaria, 7(4), 25-38. doi: https://doi.org/10.4067/S071850062014000400004

Sandberg, J. (2000). Understanding Human Competence at Work: An interpretative approach. Academy of Management Journal, 43(1), 9-25.

Schermelleh-Engel, K., Moosbrugger, H., \& Müller, H. (2003). Evaluating the fit of structural equation models: Tests of significance and descriptive goodness-of-fit measures. Methods of Psychological Research Online, 8(2), 23-74.

Segura, C. (2002). Aprender a aprender: claves para su enseñanza. Educación y Educadores, pp. $145-154$.

Tobón, S. (2013). Formación integral y competencias: Pensamiento complejo, currículo, didáctica y evaluación (4a ed.). Bogotá: Ecoe Ediciones. 
Vargas, J. G. (2017). Las reglas cambiantes de la competitividad global en el nuevo milenio. Red Internacional de Investigadores En Competitividad Memoria Del I Congreso, 4(1), 21002119. Retrieved from http://riico.net/index.php/riico/issue/view/14

Velásquez, C., Montgomery, W., Pomalaya, R., Vega, J., Guevara, W., García, P., \& Díaz, G. (2009). Habilidades sociales y filosofía de vida en alumnos de secundaria con y sin participación en actos violentos de Lima Metropolitana. Revista de Investigación En Psicología, 12(1), 69-82. doi: https://doi.org/10.15381/rinvp.v12i1.3781

Villarroel, V., \& Bruna, D. (2014). Reflexiones en torno a las competencias genéricas en educación superior: Un desafío pendiente. Psicoperspectivas, 13(1), 23-34.

Zavala, M., Valadez, M., \& Vargas, M. del C. (2008). Inteligencia emocional y habilidades sociales en adolescentes con alta aceptación social. Electronic Journal of Research in Educational Psychology, 6(2), 321-338.

Nota: La presente investigación fue financiada por la Universidad del Bío-Bío (FDD 2017-19). 